\title{
Circulating bile acid levels direct sex-differences in liver cancer development
}

\section{Megan E. Patton ${ }^{1}$, Sherwin Kelekar ${ }^{1}$, Lauren J. Taylor ${ }^{1}$, Rhishikesh N Thakare $^{2}$, Sung Hwan Lee ${ }^{3,4}$, Yazen Alnouti ${ }^{2}$, Zeynep Madak-Erdogan ${ }^{5}$, Ju- Seog Lee ${ }^{3}$, Milton J. Finegold ${ }^{6}$, and Sayeepriyadarshini Anakk ${ }^{1, \S}$}

$\S$ Corresponding author

${ }^{1}$ Department of Molecular and Integrative Physiology, University of Illinois, Urbana-Champaign, Urbana, IL 61801, ${ }^{2}$ Department of Pharmaceutical Sciences, College of Pharmacy, University of Nebraska, Omaha, NE 68106, ${ }^{3}$ Department of Systems Biology, The University of Texas MD Anderson Cancer Center, Houston, TX 77030, ${ }^{4}$ Department of Surgery, Yonsei University College of Medicine, Seoul, Republic of Korea, ${ }^{5}$ Department of Food, Science and Human Nutrition, University of Illinois, Urbana-Champaign, Urbana, IL 61801, ${ }^{6}$ Department of Pathology, Baylor College of Medicine, Houston, TX 77030

Conflict of Interest: None declared. 


\begin{abstract}
Hepatocellular carcinoma (HCC) is the most common liver cancer with a higher incidence in males. Here, we report that the spontaneous HCC development subsequent to the deletion of both Farnesoid X Receptor and Small Heterodimer Partner (DKO) mimics the sex-specific incidence seen clinically. In female DKO mice, we find lower levels of circulating bile acids (BA) and show that BAs can co-opt the estrogen axis to auto-regulate their homeostasis and amino acid metabolism. These regulations are lost when female mice are ovariectomized. Conversely, increasing serum BA levels is sufficient to promote tumorigenesis in female livers. To examine the translational relevance, we mined the transcriptomic signatures corresponding to that of female mice and found that it correlated well with those of low-grade tumors and associated with better outcomes for HCC patients. We demonstrate that decreasing enterohepatic recirculation of BAs using a resin dramatically reduced the liver cancer burden in male mice. These results uncover that sexual dimorphism in liver cancer incidence is linked to sex-differences in the handling of circulating BA concentrations and that lowering it might alleviate liver cancer.
\end{abstract}




\section{Significance}

We demonstrate that serum BA concentration facilitates sex-differences in tumor burden, with reduced BA levels resulting in lower HCC risk in female mice.

Increasing or decreasing circulating BA levels, promotes or alleviates HCC risk, respectively. We show that the sex-specific gene profiles identified in the DKO mice model of HCC correlate directly with patient outcomes.

\section{Keywords}

Bile acid metabolism, Cholestyramine, Sex difference, Liver cancer, Amino acid metabolism, Estrogen axis 


\section{Introduction}

Liver cancer is a leading cause of cancer-related deaths, has diverse etiologies, and displays sex-difference with reduced risk in females compared to males $(1,2)$. Since current therapies for liver cancer fall short, we posited that understanding molecular mechanisms functioning in the female livers might identify new therapeutic targets. Earlier studies have reported the role for sex hormones $(3,4)$, transcription factors FoxA1/A2 (5), and cytokine II6 signaling (6) in regulating the sex difference in Hepatocellular carcinoma (HCC), however, the role of metabolic pathways remains poorly understood.

Rewiring of cellular metabolism enables tumor cells to maintain viability and grow disproportionately (7). We examined bile acid metabolism, a function unique to the liver, since we previously showed that excessive accumulation of bile acids (BAs) is sufficient to cause spontaneous hepatic tumorigenesis $(8,9)$. BAs are cholesterol metabolites (10-12) that act as endogenous ligands for the nuclear receptor, Farnesoid X Receptor (FXR) $(13,14)$, and the G-protein coupled receptors, TGR5 (15) and S1PR (16).

Fxr and Small Heterodimer Partner (Shp) are known to negatively regulate BA synthesis (17), and the combined deletion of $\mathrm{FXr}^{--}$, Shp ${ }^{-/-}$double knockout (DKO) in mice leads to disproportionate accumulation of BAs in their serum (9). However, we found that DKO female mice not only have significantly lower BA concentration, but also exhibit a more hydrophilic serum BA composition than DKO males. This was intriguing, since the mice are genetically identical. 
BAs have been shown to promote liver tumorigenesis $(8,18)$; however their role in controlling the sexual dimorphism of HCC incidence is unknown. Therefore, we investigated if the sex differences in serum BA levels protect the DKO females from developing HCC.

We initially evaluated if estrogen signaling can regulate BA homeostasis in the absence of its canonical regulators, Fxr and Shp. Next, we analyzed the transcriptome of both sexes of WT and DKO livers. Further scrutiny of potential mechanisms revealed that in addition to maintaining lower serum BA levels, DKO female livers also restrict availability of amino acids that are necessary for proliferation. We then tested the relevance of DKO specific gene signatures in clinical cohorts. Finally, we determined if altering serum BAs is sufficient to offset the sex-differences observed in liver cancer risk.

\section{Results}

$\mathrm{FXr}^{\prime-}$, Shp ${ }^{--}$double knockout (DKO) mice phenocopy clinical features of human $H C C$

Despite loss of the primary BA homeostatic machinery, one-year-old DKO female mice did not develop liver tumors, unlike the DKO male livers, which developed well-defined adenomas along with robust steatosis, and fibrosis that progress to HCC. Instead, DKO females showed modest fat accumulation and mild fibrosis (Fig. 1A-F). Even at six months of age, female livers were smaller and displayed reduced hepatic fibrosis compared to males (SI appendix, Fig. S1). Sex-difference in tumor burden was visible and the liver to body weight 
ratios of DKO males was significantly higher than DKO females (Fig. 1G). Serum ALT and AST were elevated in the DKO animals compared to WT (Fig. 1H), which corroborates the known cholestatic phenotype of the mice. We found that the serum BA concentration in DKO females, even though higher than WT, was approximately half of that of the DKO males (Fig. 1I, SI appendix, Table. S1).

Based on this result, we questioned if mechanisms that regulated BA homeostasis influenced the sexually dimorphic incidence of liver cancer. To address this, we analyzed the expression of BA synthesis, transport, and metabolism in both sexes of DKO mice. Consistent with Fxr and Shp deletion that results in the loss of negative feedback on BA biosynthesis, both sexes of DKO mice have significantly higher expression of Cyp7a1 and Cyp8b1 genes that are involved in classical BA synthesis (Fig. 2A). On the other hand, Cyp27a1, which initiates alternative BA synthesis, was increased in a femalespecific manner (Fig. 2A).

Next, we examined if BA transport was responsible for the lower circulating levels in female mice. Hepatic transcript levels of the key BA efflux pump, Bile salt export pump (Bsep, $A b c b 11)$ were reduced in both sexes of DKO mice, consistent with loss of $F x r$ (19) (Fig. 2B). Expression of canalicular efflux transporters, Abcb1 (Mdr1) and Abcc2 (Mrp2), were unchanged (Fig. 2B). But the BA uptake transporter, Sodium taurocholate co-transporting polypeptide (Ntcp, Slc10a1) was reduced in females (Fig. 2B), which corroborated well with the previous results that estradiol can downregulate Slc10a1 expression (20). Although circulating bile acids levels in serum were lower in female DKO mice 
compared to DKO males, no difference in their hepatic BA concentrations was noted (Fig. 2D).

We then investigated the expression of genes involved in BA sulfation-a modification that can reduce entero-hepatic recirculation and promote excretion (21). Both WT and DKO females displayed dramatic induction of hepatic Sult2a1 (Fig. 2C), which is known to be female-specific (22). We tested if increased BA sulfation may contribute toward the reduced serum BA pool in females. Analysis of BA composition revealed that the pool of serum BAs in DKO females was more hydrophilic (less cytotoxic) and had a higher proportion of sulfated to total BAs than DKO males (Fig. 2E-F, H) (SI appendix, Table S1).

So, we measured urinary excretion of BAs, as it is an important route of excess BA elimination during cholestasis (23). Urine BA levels were higher in males, consistent with a larger circulating BA pool than in DKO females (SI appendix, Table S2). Despite higher hepatic Sult2a1 expression, DKO females excreted a smaller percent of it in their urine (SI appendix, Table S2). Nevertheless, urinary BA composition revealed that female mice excreted a greater proportion of toxic secondary BAs, which are known tumor promoters (24), whereas males excreted more primary BAs (Fig. 2G, I). These data suggest that the reduced $\mathrm{BA}$ concentration, along with differences in BA composition, may contribute to lower cancer incidence in female livers.

To understand how DKO mice handle excess BAs in the absence of their canonical signaling axis, we fed them 1-week of $1 \%$ Cholic Acid (CA)-enriched diet. Most $(7 / 8)$ of the DKO males died by day five, whereas all DKO female mice 
survived (8/8). While weight loss occurred in both sexes, DKO males lost slightly more than the females and accumulated more hepatic BAs when fed with the CA $\operatorname{diet}(\mathrm{SI}$ appendix, Fig. S2A-B). By day four of the diet, DKO female mice were slow but mobile, whereas, DKO males appeared moribund compared to WT animals (SI appendix, video file 1). Surprisingly, DKO mice display significant suppression of Cyp7a1 transcript levels, but the other BA synthesis genes remain unchanged (SI appendix, Fig.S2C).

Upon further examination, we found that DKO male livers displayed an increase in Estrogen receptor $\alpha$ (Era) expression on day four of the CA-diet (SI appendix, Fig. S2D). Although DKO females do not show change in Era expression at the four-day timepoint, they showed a robust increase a week after CA-diet, suggesting a temporal regulation (Fig. 3B, SI appendix, Fig. S2D). We posit that the increase in Era expression may fall short in males since they do not have ample estrogen to activate the signaling. Conversely, in females this signaling may protect them from accumulating excess BAs in the absence of FXR and SHP. To test the role for estrogen signaling in regulating BA homeostasis in DKO animals, we compared gene expression between sham and ovariectomized (OVX) female mice (Fig. 3A). As expected, OVX resulted in reduction of basal hepatic Era expression in both WT and DKO mice (Fig. 3B). Similarly, OVX was sufficient to reduce the basal levels of Cyp7a1 (Fig. 3C), and Cyp8b1 (Fig. 3D), two crucial genes responsible for BA synthesis, and Sult2a1, a gene responsible for BA sulfation and excretion in the DKO female livers (Fig. 3E). WT female mice showed CA-mediated suppression of BA synthetic genes 
that is consistent with intact FXR signaling, which is maintained after OVX.

However, DKO+OVX mice lack both Fxr and estrogen signaling and were unable to suppress $B A$ synthetic genes or induce Sult2a1 expression when challenged with CA-diet (Fig. 3E). This result led us to question if ERa played a direct role in BA regulation. We examined ERa occupancy using three independently published hepatic ERa cistrome studies (25-27) and identified ERa binding sites at the transcription start sites of Cyp7a1, and Cyp8b1, and within the Sult2a1 gene (Fig. 3F). These results revealed the importance of estrogen signaling in regulating BA homeostasis.

\section{Sex-specific metabolic programs regulate tumorigenesis}

To further explore transcriptional mechanisms that contribute to the sex differences in the incidence of hepatic tumorigenesis, we analyzed one-year-old male and female livers. DKO males and females displayed striking differences in their hepatic gene expression profile (SI appendix, Fig. S3A) with DKO males showing enrichment of endoplasmic reticulum stress, unfolded protein response, and immune function (SI appendix, Fig. S3B-C). Additional network analyses revealed interactions between drug metabolism, inflammation, Erk signaling, and steroid metabolism in DKO males (SI appendix, Fig. S3D) (28). Conversely, DKO females displayed pathway enrichment of steroid metabolism and clustering of lipid, glucose, amino acid and steroid metabolism, along with increased sulfotransferase activity (SI appendix, Fig. S3E). Next, we parsed the sexspecific upregulated gene sets to identify unique transcription factor motifs. 
Overrepresented motifs in DKO males include AR, FoxA1, FoxA2, NRF2 and PPARy, which correlate with tumor promoting functions (SI appendix, Table S3) whereas in DKO females, FoxO1, cMyc, and ERa (SI appendix, Table S4) were some of the dominant motifs.

As we focused on the sex differences, we were piqued by the unique overrepresentation of amino acid metabolism gene ontology observed in DKO female livers (Fig. 4A). Amino acids play a central role in tumorigenesis $(29,30)$, and removal of amino groups occurs primarily via the urea cycle, which is catalyzed by five enzymes, carbamoyl phosphate synthetase (CPS1), ornithine transcarbamylase (OTC), argininosuccinate synthetase (ASS1), argininosuccinate lyase (ASL), and arginase (ARG1) (Fig. 4B). We examined and found robust induction of genes responsible for the flux of glutamine, arginine and asparagine into the urea cycle in DKO female livers relative to males (Fig. 4B).

To validate the observed gene expression profile, we analyzed liver metabolites in the DKO animals. Compared to the males, DKO female livers had a significant increase in urea and urea cycle intermediates including citrulline, ornithine, glutamine and arginine (Fig. 4C). Consistent with this data, ammonia, which is removed via the urea cycle, was lower in DKO female livers (Fig. 4D). However, WT mice did not exhibit any sex differences in these metabolites (SI appendix, Fig. S4A).

BAs have recently been shown to promote ureagenesis in an FXRdependent manner (31). Intriguingly, DKO mice have high BAs, however, they 
lack FXR. Our experiments indicated that ER $\alpha$ can offset the loss of FXR in regulating BA homeostasis (Fig. 3), therefore, we extended this logic and examined if estrogen signaling can also regulate ureagenesis. Depleting endogenous estrogen signaling by OVX was sufficient to reduce expression of many of the urea cycle genes, including those that code for rate-limiting enzymes Cps1, Asl1, and Arg1 in DKO females (Fig. 4E). Further, these genes were also decreased in WT post-OVX, indicating that endogenous estrogen signaling is important to maintain basal expression of urea cycle genes (SI appendix, Fig. S4B). G/s2 showed differential regulation after OVX; it was reduced in DKO (Fig. 4E) but was induced in WT mice. Furthermore, estradiol treatment led to Era recruitment to the transcription start site of Cps1, Asl, and Arg1, and intergenic region of $\mathrm{Gls} 2$ (Fig. 4F), indicating a direct role for estrogen to modulate urea synthesis. Supplementation of BAs in the diet rescued As/ and Ass transcript levels in WT, but not in DKO females post-OVX (SI appendix, Fig. S4B, Fig.4E). This was not entirely surprising, since DKO mice inherently have excessive BAs. Taken together, these results show that BAs and estrogen signaling coordinate to maintain amino acid metabolism.

We then asked if BA levels increased when endogenous estrogen axis is depleted, and if it would promote liver cancer in DKO females. To address this, we ovariectomized DKO female mice, aged them to one-year, and examined their livers. We found that OVX (Fig. 5A) not only promoted tumorigenesis in DKO female livers, but also increased the circulating levels of BAs (Fig. 5B-C). Next, we ascertained if the protection seen in female livers against cancer 
incidence is due to lower serum BAs. We fed DKO female mice with 3,5diethoxycarbonyl -1,4-dihydrocollidine (DDC) diet, which is well-known to cause biliary disease and elevate serum BA concentrations. We found that doubling the serum BA levels was sufficient to cause development of large tumors in DKO female livers (Fig. 5D-F). Upon examination of BA composition, we found that the DDC diet decreased the overall percentage of UDCA and altered the proportions of CA and MCA in serum and liver (Fig 5G, SI appendix, Table S5 and SI Dataset 1).

We further analyzed and found that DDC-fed DKO female livers displayed suppression of urea synthesis genes, Cps1, Ass, Asl and Gls2 with their expression being reduced to that of DKO males except for Cps1 (SI appendix, Fig.S5). This result indicates that ureagenesis is dampened during hepatic carcinogenesis in females.

These experiments establish high BA concentration is crucial for tumorigenesis, and that female liver adapts estrogen signaling to constrain cellular proliferation by decreasing BA levels and nitrogen availability.

\section{DKO HCC mouse model is clinically significant}

We analyzed the WT and DKO murine transcriptomic signatures and compared these to five separate clinical HCC cohorts $(32,33)$. Using class prediction, patient samples were sorted based on their similarity to specific oneyear-old DKO signatures (SI appendix, Fig. S6, SI Dataset 2). Notably, Fxr and Shp transcripts are downregulated in samples of human HCC (Fig. 6A-B). 
We analyzed data from about 1000 patients and found that $491(\sim 44 \%)$ showed similar transcriptomic signature to that of DKO male mice and displayed lower overall survival (OS), with no difference in recurrence-free survival (RFS) (Fig. 6C, SI Dataset 2), compared to patients who did not have the DKO signature. On the other hand, $609(\sim 54.71 \%)$ patients that showed DKO female-specific signature had a better overall and recurrence-free survival (Fig. 6D, SI Dataset 2).

Apart from the entire transcriptome, we also evaluated the significance of estrogen metabolic targets and urea cycle genes that were induced in DKO female livers. Patients who expressed the DKO female estrogen signature (34), or the DKO-urea cycle signature had better clinical outcomes (Fig. 6E-F, SI dataset 2) than those patients who did not. Importantly, computational prediction scores (BCCP score 1 represents complete match, BCCP score 0 represents no match) using all of the patient samples revealed that the DKO male signature match the later stages (2-5), whereas the DKO female-specific signature match with initial stage 0 of liver cancer (Fig. 6G, SI Dataset 2). These findings indicate that the sex-specific transcriptomic signatures obtained from the DKO mouse model may help predict clinical outcomes of HCC patients.

Decreasing serum BA concentrations in DKO males is sufficient to reduce liver cancer risk

Finally, we investigated if we can prevent liver tumor growth in DKO male mice by reducing the serum BA concentration using cholestyramine (CHR), a 
resin that inhibits BA recirculation. Nine-month-old DKO male mice were fed a $2 \%$ CHR-containing diet, since by this age, they have already developed tumor nodules. The CHR diet was continued until one year of age, mimicking a therapeutic intervention. DKO males fed chow exhibit severe hepatic tumorigenesis, whereas CHR-fed DKO males have a drastically lower tumor burden with only small liver nodules and are protected from developing aggressive carcinomas (Fig. 7A-D). Histological analysis revealed that CHR treatment lowered the number of nodules and dysplastic changes, but increased steatosis in DKO males (Fig. 7C).

As expected, $\mathrm{CHR}$ diet led to a $50 \%$ reduction in serum BA levels in males (Fig. 7E), which is comparable to that of chow-fed DKO female mice. The serum and hepatic BA composition analysis revealed significant changes with increases in both taurocholate and taurochenodeoxylate/taurodeoxycholate and decreases in total MCA upon CHR treatment (Fig. 7F, SI appendix, Table S6 and SI Dataset S1). Additional unbiased cluster analysis of serum BA composition between the all cohorts of DKO mice revealed that the BA profiles of CHR-fed DKO male mice grouped with DKO females (SI appendix, Fig. S7A), demonstrating that both circulating BA concentration and composition are crucial for liver cancer development. 


\section{Discussion}

In this study, we investigated if sex differences seen in BA homeostasis is responsible for the sexual dimorphism observed in liver cancer incidence. Using a genetic mouse model that disrupts BA homeostasis, we demonstrate that there is a threshold of circulating BA levels necessary to drive tumorigenesis. Unlike female DKOs, male DKO mice have double the concentrations of circulating BAs, which is sufficient to cause liver cancer. Further, DKO female animals maintained a hydrophilic serum BA pool and exhibited differential urine BA composition relative to DKO males (Fig. 2E-H). Consistent with our studies, an elevated BA pool has been reported in males compared to females in clinics (35).

We next considered if the estrogen axis contributes towards the observed sex differences in BA homeostasis. OVX led to reduced basal gene expression of Cyp7A1 and Sult2a1. When additionally challenged with CA, DKO+OVX mice lost their ability to suppress BA synthesis genes, revealing an important role for estrogen signaling in controlling BAs. Moreover, DKO mice that were aged to a year after OVX displayed not only higher serum BA concentrations that were comparable to that of DKO males, but also developed liver cancer.

Our transcriptomic analyses revealed that female DKO livers expressed higher ureagenesis genes compared to male livers, and that this expression profile was reversed either by increasing BA levels or by OVX in the females (Fig.4E). Thus, female DKO livers may divert glutamine and arginine away from de novo pyrimidine synthesis. Cancer cells suppress urea cycle and patients with urea cycle enzyme deficiencies have a 200x greater HCC incidence, highlighting 
the importance of this pathway in hepatic tumorigenesis (36-38). Moreover, recent data revealed that BAs promote amino acid catabolism (31), which substantiate that BAs act as a central node in liver cancer development.

To investigate if reducing serum BA levels could be a possible therapeutic approach, we used a generic BA binding resin, cholestyramine $(\mathrm{CHR})$, in DKO male mice and found a dramatic protective effect against HCC. When enterohepatic recirculation of BA is decreased with $\mathrm{CHR}$, Cyp7a1 expression typically increased, leading to increased hepatic BA synthesis (39). However, we found that long-term CHR feeding in DKO mice reduced expression of Cyp7a1 but increased Cyp8b1 expression (SI appendix, Fig. S7B). CYP8B1 helps maintain a hydrophilic composition of BAs and is downregulated in HCC (40), which implies that BA composition may become hydrophobic during hepatic tumorigenesis. Consistent with this data, expression of Cyp8b1 was reduced in DKO female livers that developed tumors subsequent to DDC-diet (SI appendix, Fig. S7C).

The genetics of the DKO mouse model hampers the gut-liver cross communication that is orchestrated by the intestinal Fxr and fibroblast growth factor 15/19 (Fgf15/19). Nevertheless, the reintroduction of FXR in the intestine of FXRKO (41) as well as long-term FXR activation, and new analogues of Fgf15/19 have all been shown to be protective against liver cancer (42). Further, other modalities such as blocking BA uptake into the intestine by apical sodium bile acid transporter $(A s b t)(43,44)$ and reuptake into the portal circulation by Ost $\alpha / \beta(45,46)$ can be utilized to reduce BA reabsorption from the gut. 
Finally, estrogen and androgen signaling have been linked to protect and predispose to liver cancer $(5,6,47,48)$ Our results indicate that one of estrogenmediated protective mechanisms includes the regulation of BA homeostasis. Recent findings have shown that estrogen therapy in post-menopausal women significantly reduce their risk for developing HCC (49) underscoring its relevance.

Overall, our findings demonstrate a fundamental role for BA metabolism in facilitating the sex-differences in HCC incidence. Thus, therapies that modulate the concentration and composition of BAs will be beneficial to combat HCC. 


\section{Methods}

\section{Experimental Design}

This study was designed to elucidate the role for bile acids (BAs) in the sexually dimorphic incidence of HCC and assess the therapeutic benefits of reducing circulating $\mathrm{BA}$ levels on $\mathrm{HCC}$ development. $\mathrm{Fxr}^{-1-}$, Shp ${ }^{-/-}$(DKO) mice were maintained on a C57BL/6 background at the University of Illinois, UrbanaChampaign. Male and female mice were sacrificed at 8-12 weeks, or 6 and 12 months after birth. Mice were housed on a standard 12-hour light/dark cycle and fed normal chow and water ad libitum. To study estrogen signaling, bilateral ovariectomies were performed on WT and DKO females at 8-10 weeks old, followed by one week of recovery, and one week of chow or $1 \%$ CA (cholic acid) -supplemented chow. $2 \%$ Cholestyramine-supplemented chow was fed to 9month-old DKO male mice for a period of 3 months while $0.1 \%$ DDC $(3,5-$ diethoxycarbonyl-1, 4-dihydrocollidine) was fed to 10-month-old DKO female mice for 3 months. Urea cycle studies were performed on mice after overnight fasting. All studies were carried out as outlined in the Guide for the Care and Use of Laboratory Animals prepared by the National Academy of Sciences and published by the National Institutes of Health (National Institutes of Health publication 86-23, revised 1985). For biological harvesting, mice were anaesthetized and euthanized as described by IUCAC. Tissue was flash frozen in liquid nitrogen and blood was collected and spun down for serum. 


\section{Serum Chemistry}

Blood was collected by retro-orbital bleeding and centrifugation at $8000 \mathrm{~g} \mathrm{x}$ 10 minutes to separate serum. Serum ALT and AST were measured using Infinity ALT and Infinity AST kits (Thermo Scientific). Calorimetric measurement of serum and hepatic bile acids was performed with the Total Bile Acid (NBT method) kit (Genway Biotech).

\section{Bile Acid Analysis}

Serum and urine from DKO male and female mice fed chow was analyzed for composition of bile acids and their sulfated metabolites at University of Nebraska Medical Center. Liquid chromatographic-mass spectrometry analysis was performed with a Waters ACQUITY column (Milford, MA). Bile acids and internal standards were prepared in methanol and analyzed. Serum from DKO male and female mice fed chow, DKO males fed CHR, and DKO females fed DDC was analyzed for bile acid composition at Baylor College of Medicine Metabolomics Core. Liquid chromatographic-mass spectrometry analysis was performed with a Waters ACQUITY UPLC BEH C18 column (Milford, MA). Bile acids were detected in negative mode, with L-Zeatine added to each sample as an internal standard. The hydrophobic index was calculated as previously described (50). The raw values of BA analysis are included in SI Dataset1.

\section{Metabolite Profiling}

Liver tissue was weighed and sonicated in $70 \%$ methanol, followed by centrifugation. Supernatant was flash frozen and used for subsequent LC-MS 
analysis for urea cycle metabolites. Tissue lysate was used for BCA assay to determine protein concentration of each sample. All metabolite concentrations were normalized to protein concentration of the lysate.

\section{Histology}

Liver samples were fixed in $10 \%$ neutral-buffered formalin and used for hematoxylin and eosin, and sirius red staining.

\section{RNA extraction and quantitative PCR analysis}

Total RNA from liver was prepared according to the TRIzol (Invitrogen) protocol. cDNA was synthesized using Maxima Reverse Transcriptase (Thermo Scientific), according to manufacturer's protocol. q-RTPCR was performed on an Illumina Eco Platform. For qRT-PCR analysis, 50 ng of cDNA was added to each SYBR green-based reaction. qRT-PCR primers are provided in Table S5.

\section{Microarray}

Microarray was performed by Dr. Ju-Seog Lee's laboratory at the MD Anderson Cancer Center. Liver samples from 12-month-old male and female WT and DKO mice were collected and snap-frozen. Total RNA was isolated, labeled, and hybridized to BeadChip Array MouseWG-6 (Illumina). Bead chips were scanned with an Illumina BeadArray Reader. Microarray analysis was performed on the Illumina mouseRefseq-8 Expression platform. Upregulated gene sets were generated from genes with fold change $>1.3(p<0.0001)$ compared to control group (i.e. DKO males vs. DKO females). These gene sets were then used for downstream analyses with DAVID Bioinformatics Resources 
Analysis Software and ClueGO. Detailed information on the analysis of this microarray, previously published Era ChIP seq (25-27), and clinical HCC cohorts are provided in SI appendix.

\section{Statistical Analysis:}

All statistical tests were performed using GraphPad Prism software. Data are presented as means \pm SEM. Multiple group comparisons were analyzed by one-way or two-way ANOVA with the post hoc Bonferroni test. Unpaired t-test was used for comparison between two groups. P values $\leq 0.05$ were determined to be significant, unless otherwise noted in legends.

\section{Data Availability}

The gene expression data generated and used in this publication have been deposited in NCBI's Gene Expression Omnibus and are accessible through GEO Series accession number GSE151524.

(https://www.ncbi.nlm.nih.gov/geo/query/acc.cgi?acc=GSE151524).

Acknowledgements: We thank the Systems Biology Laboratory at the University of Texas, MD Anderson Cancer Center for initial analysis and performing the microarray studies. We would also like to thank the metabolite analysis core at Baylor College of Medicine for performing bile acid composition analysis. We thank Dr. Bhoomika Mathur for initiating studies with the DKO female and DDC experiment. The authors also thank Drs. Auinash Kalsotra and Stephanie Ceman for their comments and critiques during the preparation of this 
manuscript. We would like to thank Ms. Angela Major at Texas Children's'

Hospital for histological preparation and analysis. The authors thank Dr. Lucas Li

at the Roy Carver Metabolomics Core at University of Illinois at Urbana-

Champaign for Urea Cycle metabolite analysis.

Author contributions: M.E.P., S.K., and S.A. contributed to conception, experimental design, data acquisition, analysis and interpretation, and drafting the article. L.J.T. contributed to ovariectomy studies, data acquisition, analysis, and editing of the manuscript, R.N.T. and Y.A. analyzed bile acid composition and interpretation from serum and urine, Z.M.E analyzed ER $\alpha$ ChIP-seq data, H.S.L and J.S.L performed all the HCC patient cohort analysis, M.J.F. evaluated and interpreted the liver histology.

Competing interests: None

Funding: This work was supported in part by NIDDK grant, R01 DK113080 (S. A), Research Scholar Grant ACS 132640-RSG (S.A) and UIUC start-up funds (S.A.) 


\section{References}

1. A. Forner, M. Reig, J. Bruix, Hepatocellular carcinoma. Lancet 391, $1301-$ 1314 (2018).

2. D. L. White, A. P. Thrift, F. Kanwal, J. Davila, H. B. El-Serag, Incidence of Hepatocellular Carcinoma in All 50 United States, From 2000 Through 2012. Gastroenterology 152, 812-820 e815 (2017).

3. P. Liu et al., Age-specific sex difference in the incidence of hepatocellular carcinoma in the United States. Oncotarget 8, 68131-68137 (2017).

4. K. A. McGlynn et al., Reproductive factors, exogenous hormone use and risk of hepatocellular carcinoma among US women: results from the Liver Cancer Pooling Project. Br J Cancer 112, 1266-1272 (2015).

5. Z. Li, G. Tuteja, J. Schug, K. H. Kaestner, Foxa1 and Foxa2 are essential for sexual dimorphism in liver cancer. Cell 148, 72-83 (2012).

6. W. E. Naugler et al., Gender disparity in liver cancer due to sex differences in MyD88-dependent IL-6 production. Science 317, 121-124 (2007).

7. N. N. Pavlova, C. B. Thompson, The Emerging Hallmarks of Cancer Metabolism. Cell Metab 23, 27-47 (2016).

8. S. Anakk et al., Bile acids activate YAP to promote liver carcinogenesis. Cell Rep 5, 1060-1069 (2013).

9. S. Anakk et al., Combined deletion of Fxr and Shp in mice induces Cyp17a1 and results in juvenile onset cholestasis. J Clin Invest 121, 86-95 (2011).

10. A. F. Hofmann, Chemistry and enterohepatic circulation of bile acids. Hepatology 4, 4S-14S (1984).

11. F. Kuipers, V. W. Bloks, A. K. Groen, Beyond intestinal soap--bile acids in metabolic control. Nat Rev Endocrinol 10, 488-498 (2014).

12. D. W. Russell, The enzymes, regulation, and genetics of bile acid synthesis. Annu Rev Biochem 72, 137-174 (2003).

13. D. J. Parks et al., Bile acids: natural ligands for an orphan nuclear receptor. Science 284, 1365-1368 (1999).

14. H. Wang, J. Chen, K. Hollister, L. C. Sowers, B. M. Forman, Endogenous bile acids are ligands for the nuclear receptor FXR/BAR. Mol Cell 3, 543553 (1999).

15. Y. Kawamata et al., A G protein-coupled receptor responsive to bile acids. J Biol Chem 278, 9435-9440 (2003).

16. E. Studer et al., Conjugated bile acids activate the sphingosine-1phosphate receptor 2 in primary rodent hepatocytes. Hepatology 55, 267276 (2012).

17. B. Goodwin et al., Differential regulation of rat and human CYP7A1 by the nuclear oxysterol receptor liver X receptor-alpha. Mol Endocrinol 17, 386394 (2003).

18. L. Sun et al., Bile acids promote diethylnitrosamine-induced hepatocellular carcinoma via increased inflammatory signaling. Am J Physiol Gastrointest Liver Physiol 311, G91-G104 (2016). 
19. M. Wagner et al., Role of farnesoid X receptor in determining hepatic ABC transporter expression and liver injury in bile duct-ligated mice. Gastroenterology 125, 825-838 (2003).

20. F. R. Simon, J. Fortune, M. Iwahashi, I. Qadri, E. Sutherland, Multihormonal regulation of hepatic sinusoidal Ntcp gene expression. Am J Physiol Gastrointest Liver Physiol 287, G782-794 (2004).

21. E. H. De Witt, L. Lack, Effects of sulfation patterns on intestinal transport of bile salt sulfate esters. Am J Physiol 238, G34-39 (1980).

22. Y. Alnouti, C. D. Klaassen, Mechanisms of gender-specific regulation of mouse sulfotransferases (Sults). Xenobiotica 41, 187-197 (2011).

23. I. Makino, H. Hashimoto, K. Shinozaki, K. Yoshino, S. Nakagawa, Sulfated and nonsulfated bile acids in urine, serum, and bile of patients with hepatobiliary diseases. Gastroenterology 68, 545-553 (1975).

24. S. Yoshimoto et al., Obesity-induced gut microbial metabolite promotes liver cancer through senescence secretome. Nature 499, 97-101 (2013).

25. J. Gertz et al., Distinct properties of cell-type-specific and shared transcription factor binding sites. Mol Cell 52, 25-36 (2013).

26. F. K. Gordon et al., Research resource: Aorta- and liver-specific ERalphabinding patterns and gene regulation by estrogen. Mol Endocrinol 28, 1337-1351 (2014).

27. G. Palierne et al., Changes in Gene Expression and Estrogen Receptor Cistrome in Mouse Liver Upon Acute E2 Treatment. Mol Endocrinol 30, 709-732 (2016).

28. G. Bindea et al., ClueGO: a Cytoscape plug-in to decipher functionally grouped gene ontology and pathway annotation networks. Bioinformatics 25, 1091-1093 (2009).

29. J. Zhang, N. N. Pavlova, C. B. Thompson, Cancer cell metabolism: the essential role of the nonessential amino acid, glutamine. EMBO J 36, 1302-1315 (2017).

30. A. M. Hosios et al., Amino Acids Rather than Glucose Account for the Majority of Cell Mass in Proliferating Mammalian Cells. Dev Cell 36, 540549 (2016).

31. V. Massafra et al., Farnesoid X Receptor Activation Promotes Hepatic Amino Acid Catabolism and Ammonium Clearance in Mice. Gastroenterology 152, 1462-1476 e1410 (2017).

32. J. S. Lee et al., Application of comparative functional genomics to identify best-fit mouse models to study human cancer. Nat Genet 36, 1306-1311 (2004).

33. J. S. Lee et al., A novel prognostic subtype of human hepatocellular carcinoma derived from hepatic progenitor cells. Nat Med 12, 410-416 (2006).

34. S. Della Torre et al., An Essential Role for Liver ERalpha in Coupling Hepatic Metabolism to the Reproductive Cycle. Cell Rep 15, 360-371 (2016).

35. L. J. Bennion et al., Sex differences in the size of bile acid pools. Metabolism 27, 961-969 (1978). 
36. w. b. e. Cancer Genome Atlas Research Network. Electronic address, N. Cancer Genome Atlas Research, Comprehensive and Integrative Genomic Characterization of Hepatocellular Carcinoma. Cell 169, 13271341 e1323 (2017).

37. M. Koo, G. S. Lipshutz, S. D. Cederbaum, C. Lassman, Biopsy-proven Hepatocellular Carcinoma in a 53-year-old Woman With Arginase Deficiency. Pediatr Dev Pathol 20, 517-521 (2017).

38. S. Rabinovich et al., Diversion of aspartate in ASS1-deficient tumours fosters de novo pyrimidine synthesis. Nature 527, 379-383 (2015).

39. M. Schwarz, D. W. Russell, J. M. Dietschy, S. D. Turley, Alternate pathways of bile acid synthesis in the cholesterol 7alpha-hydroxylase knockout mouse are not upregulated by either cholesterol or cholestyramine feeding. J Lipid Res 42, 1594-1603 (2001).

40. M. J. Hoenerhoff et al., Global gene profiling of spontaneous hepatocellular carcinoma in B6C3F1 mice: similarities in the molecular landscape with human liver cancer. Toxicol Pathol 39, 678-699 (2011).

41. C. Degirolamo et al., Prevention of spontaneous hepatocarcinogenesis in farnesoid $X$ receptor-null mice by intestinal-specific farnesoid $X$ receptor reactivation. Hepatology 61, 161-170 (2015).

42. R. M. Gadaleta et al., Suppression of Hepatic Bile Acid Synthesis by a non-tumorigenic FGF19 analogue Protects Mice from Fibrosis and Hepatocarcinogenesis. Sci Rep 8, 17210 (2018).

43. A. G. Miethke et al., Pharmacological inhibition of apical sodiumdependent bile acid transporter changes bile composition and blocks progression of sclerosing cholangitis in multidrug resistance 2 knockout mice. Hepatology 63, 512-523 (2016).

44. A. Rao et al., Inhibition of ileal bile acid uptake protects against nonalcoholic fatty liver disease in high-fat diet-fed mice. Sci Transl Med 8 , 357 (2016).

45. A. Baghdasaryan et al., Inhibition of intestinal bile acid absorption improves cholestatic liver and bile duct injury in a mouse model of sclerosing cholangitis. J Hepatol 64, 674-681 (2016).

46. S. M. W. van de Wiel, D. R. de Waart, R. P. J. Oude Elferink, S. F. J. van de Graaf, Intestinal Farnesoid X Receptor Activation by Pharmacologic Inhibition of the Organic Solute Transporter alpha-beta. Cell Mol Gastroenterol Hepatol 5, 223-237 (2018).

47. W. L. Ma et al., Androgen receptor is a new potential therapeutic target for the treatment of hepatocellular carcinoma. Gastroenterology 135, 947955, 955 e941-945 (2008).

48. I. Shimizu et al., Suppressive effect of oestradiol on chemical hepatocarcinogenesis in rats. Gut 42, 112-119 (1998).

49. M. M. Hassan et al., Estrogen Replacement Reduces Risk and Increases Survival Times of Women With Hepatocellular Carcinoma. Clin Gastroenterol Hepatol 15, 1791-1799 (2017).

50. D. M. Heuman, Quantitative estimation of the hydrophilic-hydrophobic balance of mixed bile salt solutions. J Lipid Res 30, 719-730 (1989). 
A

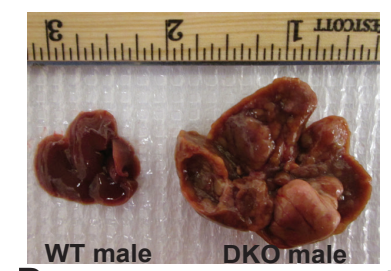

D

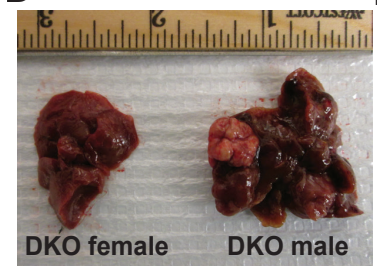

G Liver/Body Weight ratio

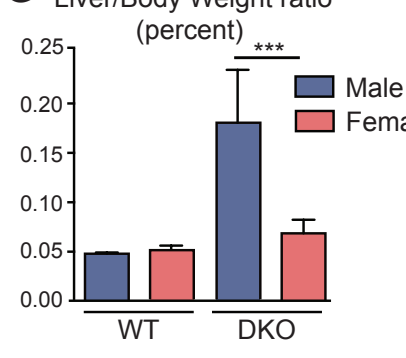

B

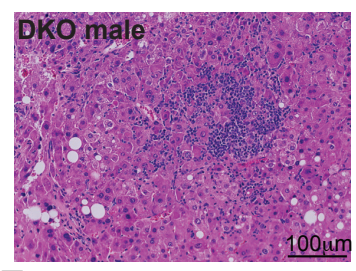

E

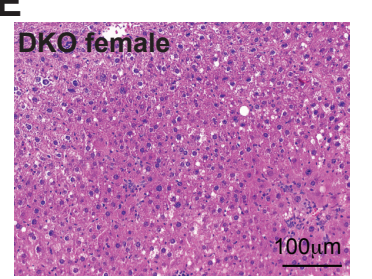

H Serum ALT

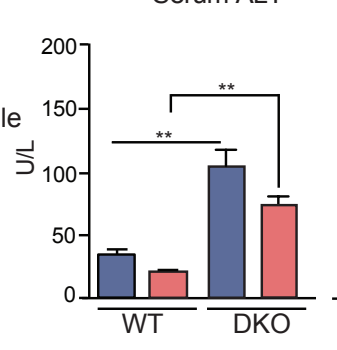

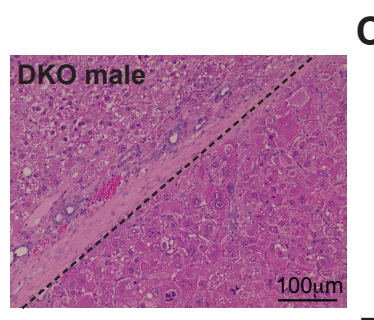

C

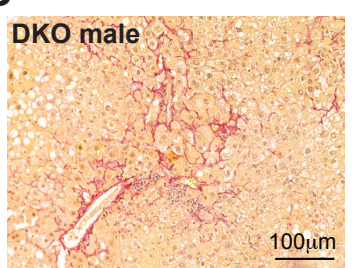

F
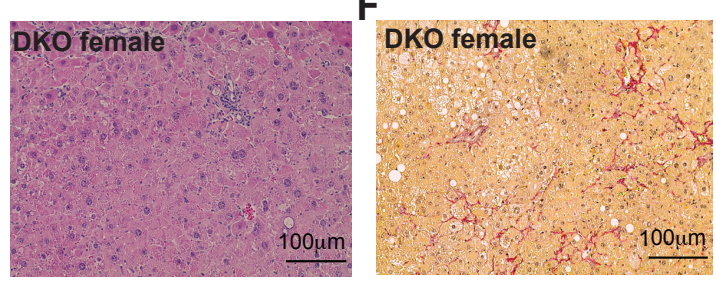

Serum Bile Acids
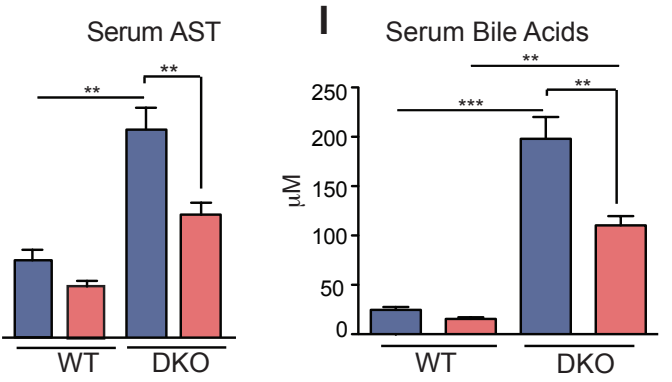

Figure 1: Fxr/Shp double knockout (DKO) mouse model recapitulates

\section{sex-difference in HCC incidence.}

(A-E) One-year-old DKO male mice developed hepatocellular carcinoma, which was not observed in age-matched wild type (WT) and DKO female mice. (B and

E) Representative H\&E stained liver sections from a (B) DKO male and (E)

female. Inflammation and injury are evident at 1 year and the dotted line separates the HCC having large nuclei and mitosis on lower right ( $\mathrm{C}$ and $\mathrm{F}$ ). Sirius red staining shows increased collagen in a perisinusoidal distribution, which is greater in the DKO males. $(G)$ Liver-to-body weight ratio was significantly higher in DKO males. Compared to DKO males, serum 
markers of liver injury, (H) AST and ALT, along with (I) serum bile acid levels were lower in DKO females. $n=5-10$ mice/group; mean \pm SEM; * $p<0.01$, ${ }^{* *} p<0.001$ compared to genotype or gender controls. One-way ANOVA with Bonferroni post hoc analysis was performed. 

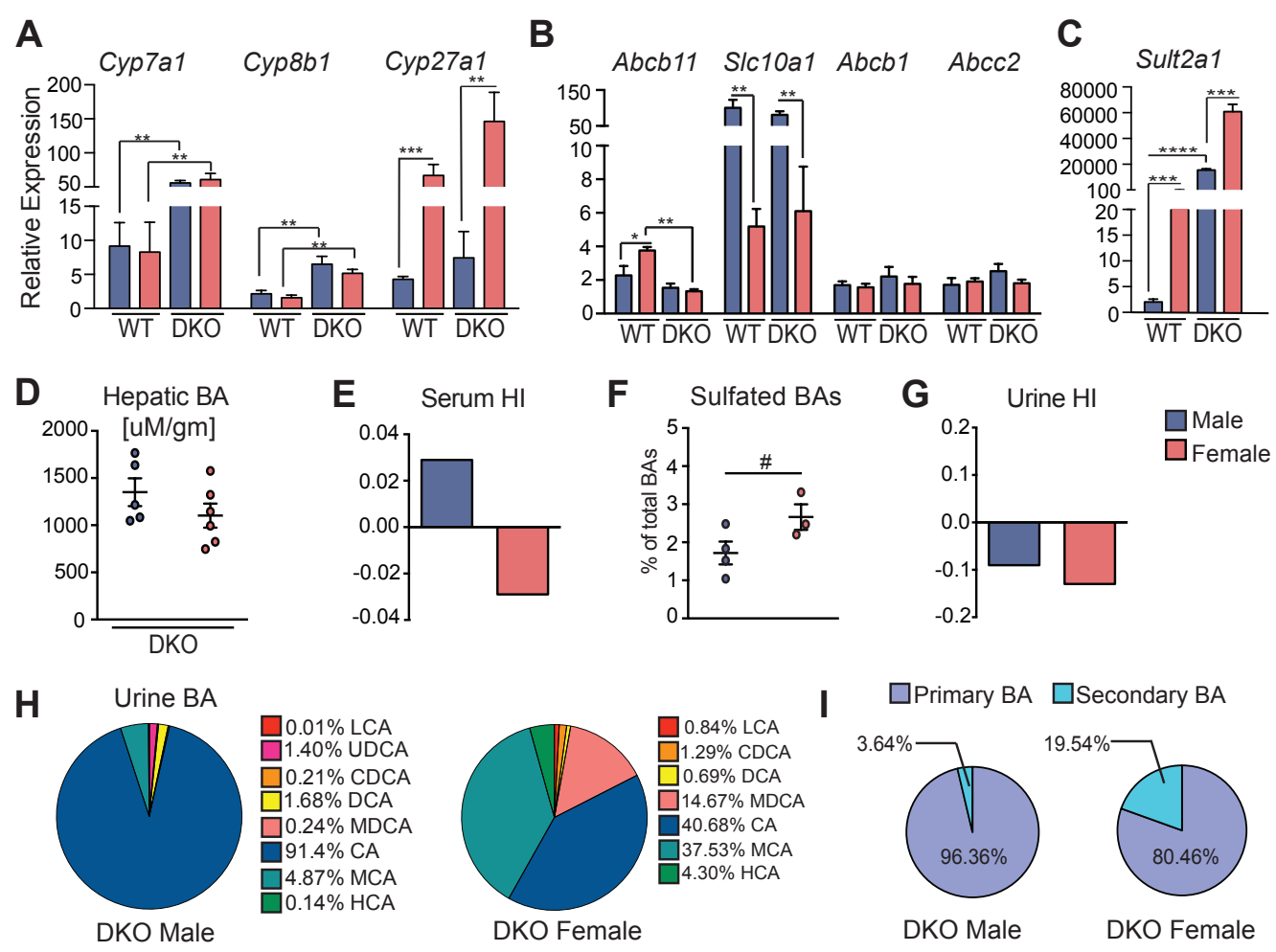

\section{Figure 2: BA composition and metabolism are differentially regulated}

\section{between the genders in DKO mice.}

(A) Hepatic mRNA expression of classical and alternative BA synthetic enzymes was analyzed in DKO and WT mice. (B-C) Expression of hepatic BA transporters and BA sulfotransferase in WT and DKO mice ( $n=5-7$ mice/group). (D) Hepatic BA concentration was unchanged between DKO males and females ( $n=5$ mice/group). (E) Calculated hydrophobicity index $(\mathrm{HI})$ of serum BA pool. (F) Percentages of sulfated BAs in DKO male and female serum. (G) Calculated hydrophobicity index $(\mathrm{HI})$ of urine BA pool. $(\mathrm{H})$ Urine BA composition was variable between sexes of DKO mice. (I) DKO females excreted a larger percentage of secondary BAs in urine compared to males. (6-month-old mice 
was used in this analysis, $n=3-4$ mice/group). Mean $\pm \mathrm{SEM}$; $\# p<0.05,{ }^{*} \mathrm{p}<0.01$, ${ }^{* *} p<0.001$ compared to genotype or gender controls. One-way ANOVA with Bonferroni post hoc was performed for gene expression analysis and one tailed t-test was performed for analyzing sulphated BAs. 

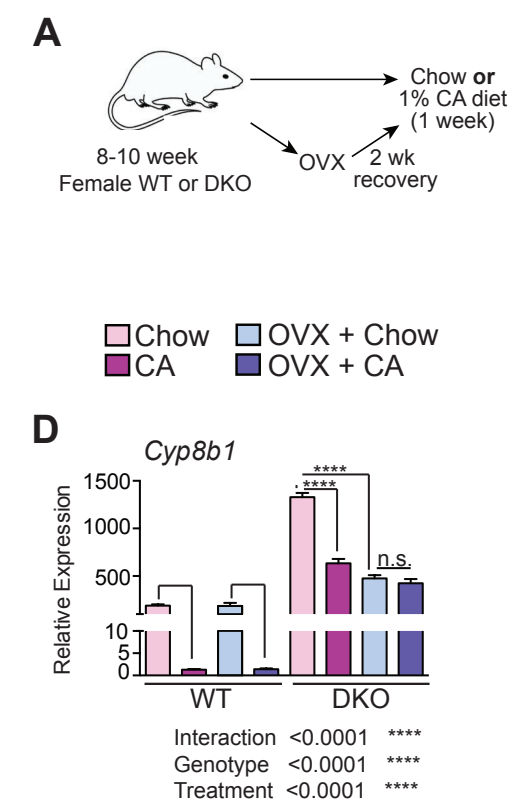

$\mathbf{F}$
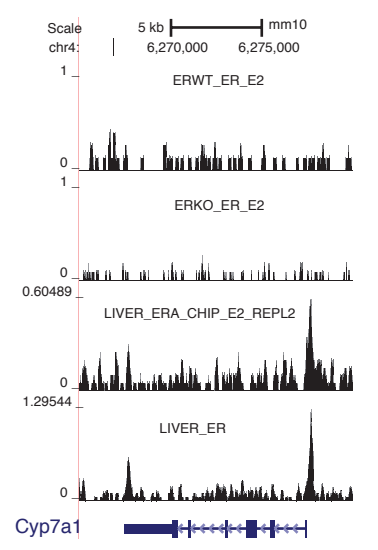

B

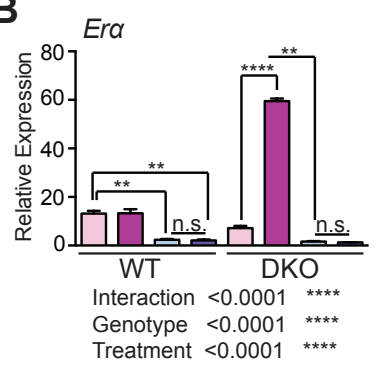

E

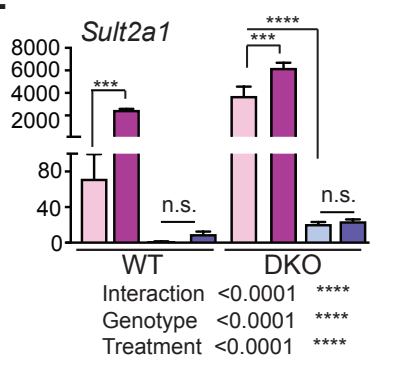

C

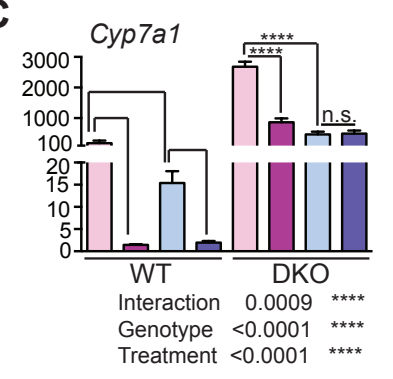

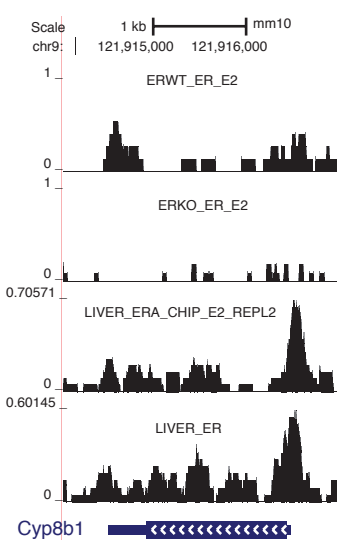

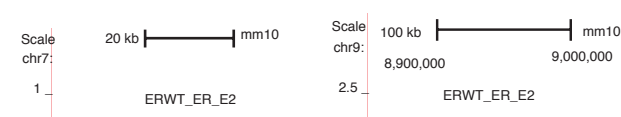

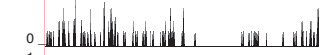
ERKO_ER_E2
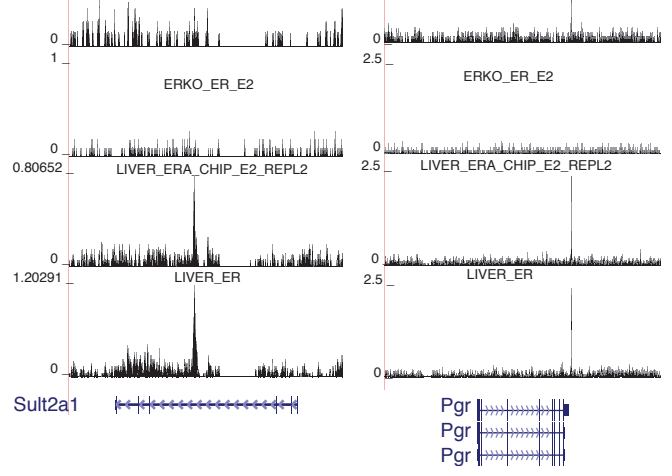

Figure 3: Estrogen signaling can regulate BA synthesis in DKO

\section{female mice.}

(A) Experimental design of chow and 1\% cholic acid (CA) diet for 1 week with or without ovariectomy (OVX). (B) Expression of hepatic Era was induced with CA diet in DKO female mice. (C) CA-mediated suppression of Cyp7a1 and (D) Cyp8b1 in WT and DKO females were lost in DKO females after OVX (E). 
Sult2a1 has greater baseline expression in DKO mice and was induced to a lesser extent upon CA challenge than WT animals ( $n=4-5$ mice/group). Mean \pm SEM; $\# p<0.05,{ }^{*} p<0.01,{ }^{* *} p<0.001$ compared to genotype or gender controls. Two-way ANOVA with Bonferroni post hoc analysis was performed. (F) Hepatic ERa cistromes, as referenced in the methods, show binding to BA synthesis and metabolism genes, Cyp7a1, Cyp8b1, and Sult2a1. Pgr, progesterone receptor binding, was used as a positive peak. Wild-type animals treated with placebo (track 1), ERKO animals treated with E2 (track 2, Palierne et al.,), and wild type mice treated with E2 (Gordon et al., track 3 and Gertz et al., track 4). 
A

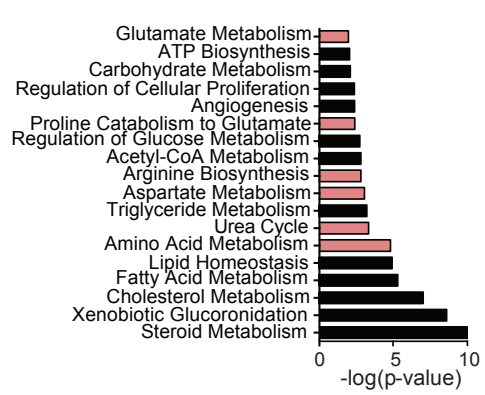

C

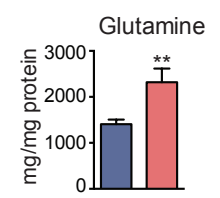

D

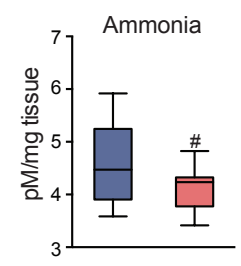

E
B
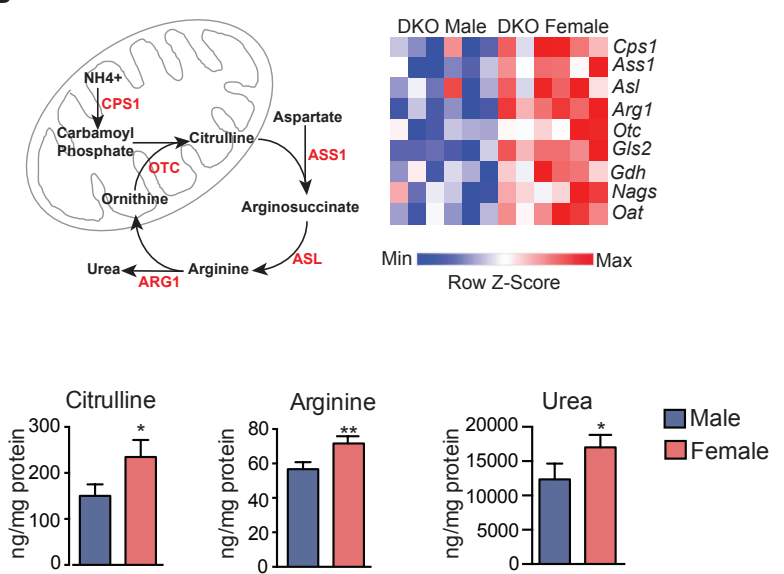

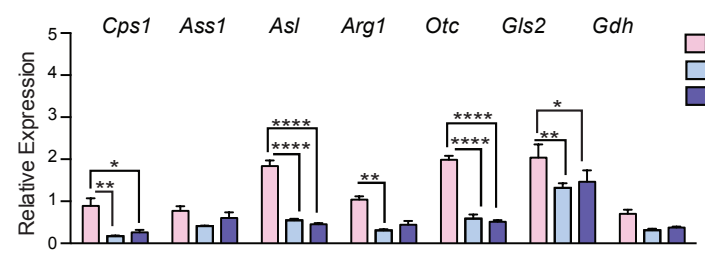

$\mathbf{F}$

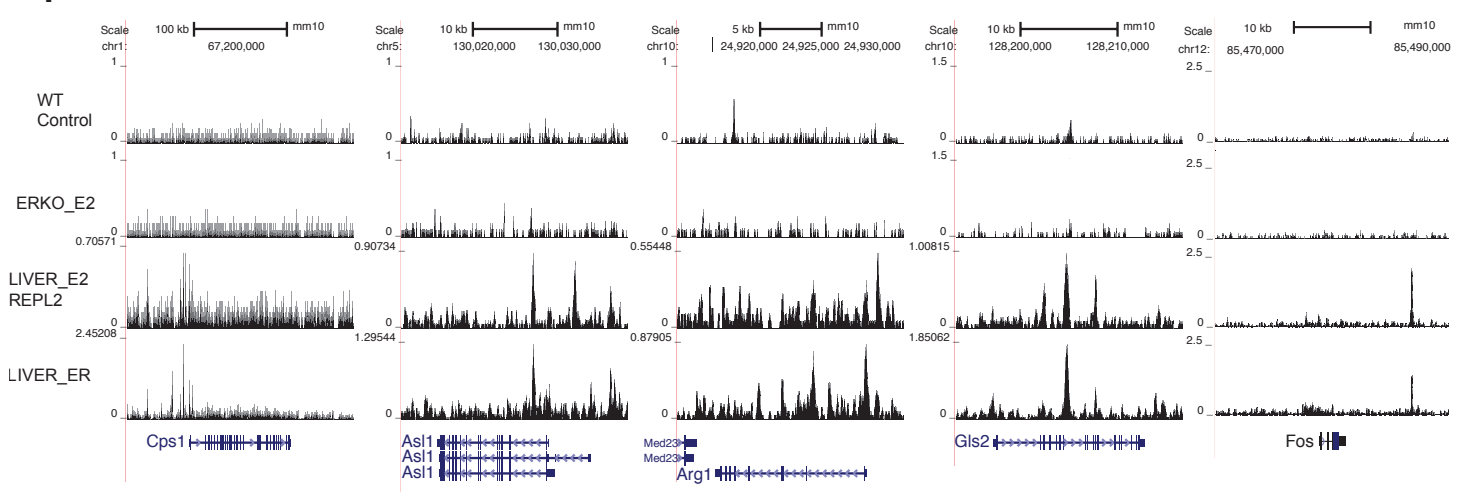

Figure 4: Amino acid metabolism and the urea cycle are controlled by the estrogen axis, particularly in the absence of Fxr and Shp.

(A) Microarray analysis was performed on both sexes of one-year-old WT and

DKO livers. Gene Ontology terms generated from a list of genes upregulated

$>1.3$ fold in DKO females compared to males $(p<0.0001)$ ( $n=6$ mice/group).

The GO terms associated with amino acid metabolism are colored in pink bars. 
(B) Schematic of the urea cycle, and heat map of urea cycle genes from the microarray ( $n=6$ mice/group). (C) LC-MS quantification of urea cycle intermediates and (D) ammonia levels in DKO livers ( $n=4-5$ mice/group). (E) Expression of urea cycle genes was reduced in DKO female mice following OVX ( $n=4-5$ mice/group). (F) ERa cistromes used for analysis are referenced in the methods and ERa binding to the Fos gene was used as a positive control. Genome tracks for urea cycle genes Cps1, Asl,1 Arg1, and Gls2 are shown. Hepatic ERa cistromes in placebo-treated wild-type mice (track 1), E2-treated ERKO mice (track 2), wild type mice treated with E2 (track 3 and 4) Mean \pm SEM; ${ }^{*} p<0.01,{ }^{* *} p<0.001$ compared to genotype or gender controls. Unpaired ttest was used for metabolite analysis and one-way ANOVA with Bonferroni post hoc was performed for gene expression analysis. 
A

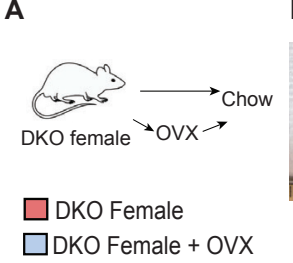

B

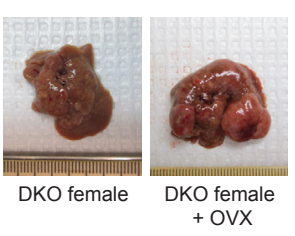

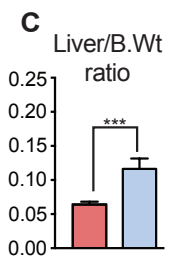

D Serum BA

E
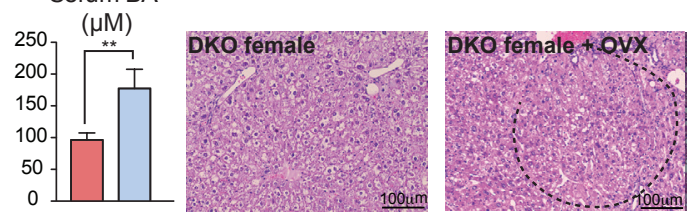

$\mathbf{F}$

G

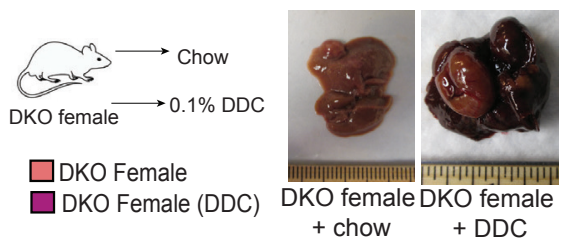

H Liver/B.Wt
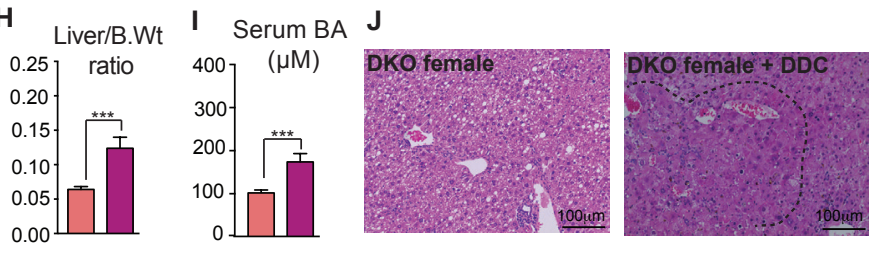

K

DKO Female +chow

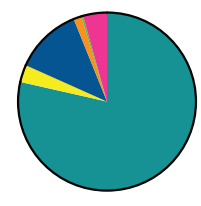

$78.53 \%$ Total MCA $12.20 \% \mathrm{~T}-\mathrm{CA}$ $1.45 \%$ T-CDCA/T-DCA $\square 0.03 \% \mathrm{HCA}$ - $4.34 \%$ UDCA $0.34 \%$ UCA 品 $\square 0.31 \%$ T-MDCA

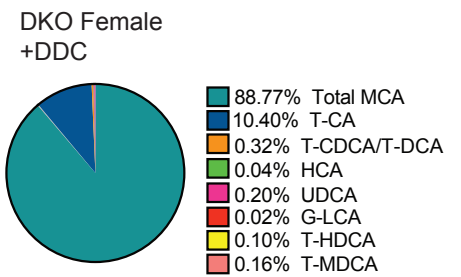

Figure 5: Female DKO livers lost their resistance to tumorigenesis upon loss of estrogen signaling and increasing serum bile acids. (A-E) DKO female mice underwent OVX surgeries at 2 months and aged to a year. OVX DKO female mice developed large liver tumors and accumulated higher serum BA levels than control DKO animals $(n=4-6$ mice/group was used). (F-G) DKO females fed a 0.1\% DDC diet for 5 months had visible tumors at one year. $(\mathrm{H}-\mathrm{I})$ Increased tumorigenesis in DKO female mice correlated with elevated liver-to-body weight ratio and serum BAs. (J) DKO female mice exhibit steatosis, cholangitis, and upon DDC treatment, develop more nodules (marked by a dotted line) and severe 
ductular reaction with bile plugs ( $n=4-7$ mice/group). (K) Serum BA composition was analyzed in chow, and DDC fed DKO female mice $(n=5-8$

mice/group). Mean \pm SEM; t-test was performed ${ }^{*} p<0.01 ;{ }^{* *} p<0.001$ compared to DKO controls. 
A

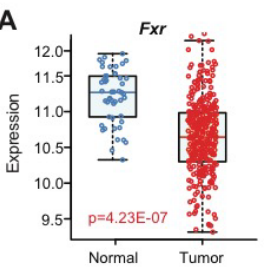

D

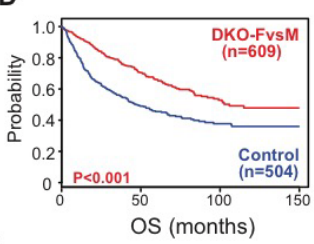

$\mathbf{F}$

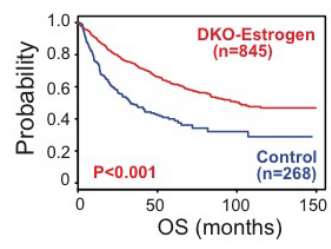

B
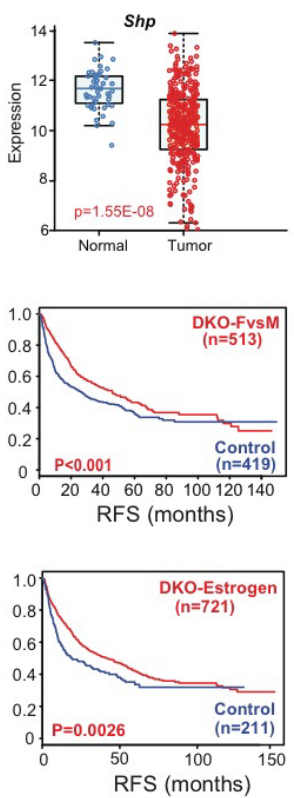

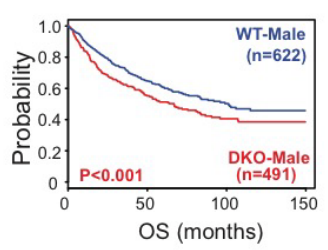

E
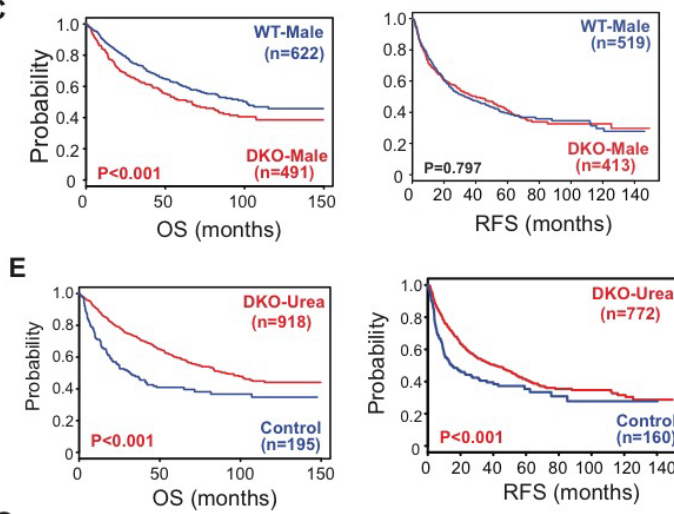

G

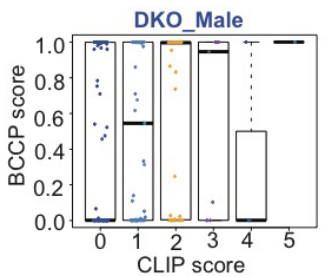

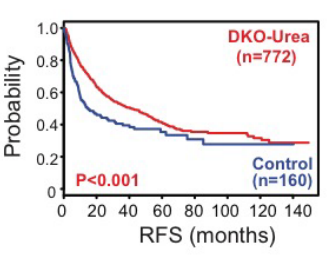

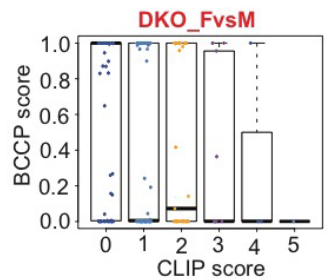

Figure 6: Gene signatures obtained from the DKO mouse model correlate well with the clinical outcomes of HCC patient samples. (A-B) Analysis of five different HCC clinical cohorts $(n=1000)$ patients reveals a reduction in Fxr and Shp transcript levels in patients with liver tumors. (C-F) Analysis of OS (Overall Survival) and RFS (Recurrence Free Survival) in patients using the gene signatures representative of male DKO mice (C), unique to female DKO mice (D) Estrogen targeted metabolic genes altered in DKO mice $(F)$, DKO-Urea cycle gene signature $(E)$. (G) BCCP prediction values for different CLIP score were evaluated for DKO male and female-specific gene sets. The ANOVA analysis for the prediction values are DKO_Male is $p<0.000269$, and DKO_FvsM is $p<0.000664$. 
A
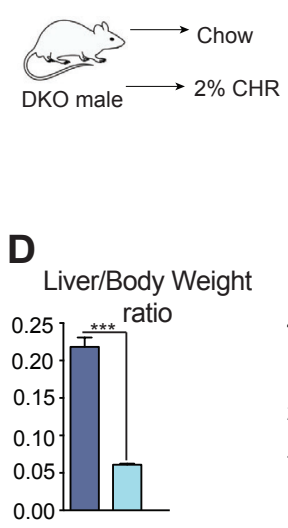

$\square$ DKO Male
B

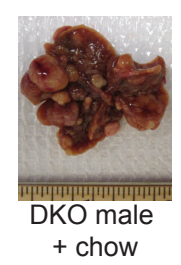

E

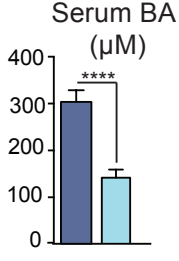

$0 \Perp 1$

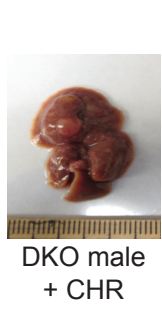

$\mathbf{F}$

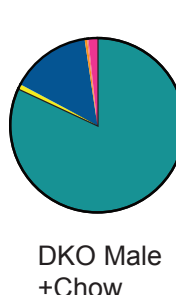

+ Chow
C

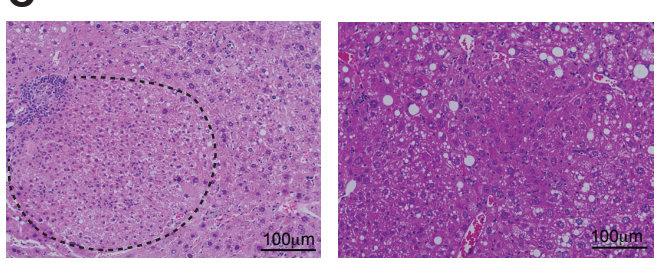

$\square 81.93 \%$ Total MCA

$\square 14.87 \%$ T-CA

$0.44 \% \mathrm{~T}$-CDCA/T-DCA
$0.02 \% \mathrm{HCA}$

$\square 1.66 \%$ UDCA

$\square 0.01 \%$ G-LCA

$\square 0.82 \%$ T-HDCA

$\square .25 \%$ T-MDCA

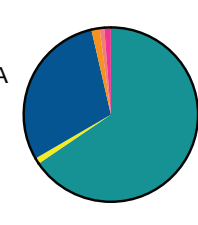

DKO Male $+\mathrm{CHR}$

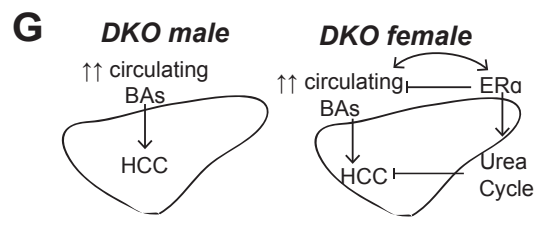

Figure 7: Treating with BA-binding resin reduces the tumor burden in DKO male mice.

(A) DKO male mice were fed a $2 \%$ cholestyramine (CHR)-enriched diet for 3 months until one year of age. Compared to chow-fed controls, (B) CHR dramatically reduced HCC burden in DKO males. (C) Histological analysis shows inflammation and tumor nodule (marked by a dotted line) in DKO male mice. CHR treatment increased steatosis, but resulted in smaller and fewer tumor nodules, as reflected in the liver weight (D). (E-F) Serum BA levels are reduced upon feeding DKO male mice CHR diet ( $n=6-7 /$ group) and their composition is shown ( $n=4-5$ mice/group). (G) Schematic showing the protective mechanisms employed against HCC development by DKO female livers. T-test was used for the analysis. Mean \pm SEM; ${ }^{*} p<0.01 ;{ }^{* *} p<0.001$ compared to DKO controls. 